\title{
Software Strategy for Robotic Transperineal Prostate Therapy in Closed-Bore MRI
}

\author{
Junichi Tokuda ${ }^{1}$, Gregory S. Fischer ${ }^{2}$, Csaba Csoma ${ }^{2}$, Simon P. DiMaio ${ }^{3}$, \\ David G. Gobbi ${ }^{4}$, Gabor Fichtinger ${ }^{4}$, Clare M. Tempany ${ }^{1}$, and Nobuhiko Hata ${ }^{1}$ \\ ${ }^{1}$ Department of Radiology, Brigham and Women's Hospital \\ and Harvard Medical School, 75 Francis St., Boston, MA 02115, USA \\ \{tokuda, ctemapny, hata\}@bwh . harvard.edu \\ ${ }^{2}$ Engineering Research Center for Computer Integrated Surgery, Johns Hopkins \\ University, 3400 N. Charles St., Baltimore, MD 21218, USA \\ $\{$ gfischer, csoma $\} @$ jhu.edu \\ ${ }^{3}$ Intuitive Surgical Inc., 950 Kifer Road, Sunnyvale, CA 94086, USA \\ Simon.DiMaio@intusurg.com \\ ${ }^{4}$ School of Computing, Queen's University, \\ 25 Union St., Kingston, Ontario, K7L 3N6, Canada \\ $\{$ dgobbi, gabor\}@cs .queensu.ca
}

\begin{abstract}
A software strategy to provide intuitive navigation for MRIguided robotic transperineal prostate therapy is presented. In the system, the robot control unit, the MRI scanner, and open-source navigation software are connected to one another via Ethernet to exchange commands, coordinates, and images. Six states of the system called "workphases" are defined based on the clinical scenario to synchronize behaviors of all components. The wizard-style user interface allows easy following of the clinical workflow. On top of this framework, the software provides features for intuitive needle guidance: interactive target planning; 3D image visualization with current needle position; treatment monitoring through real-time MRI. These features are supported by calibration of robot and image coordinates by the fiducial-based registration. The performance test shows that the registration error of the system was $2.6 \mathrm{~mm}$ in the prostate area, and it displayed real-time $2 \mathrm{D}$ image $1.7 \mathrm{~s}$ after the completion of image acquisition.
\end{abstract}

\section{Introduction}

MRI is an ideal imaging tool for prostate biopsy and local therapy due to its high sensitivity and specificity to focal prostate lesions and surrounding tissues, and its radiation safety. An MRI scanner in the operating suite allows acquisition of the MR images used to define clinical target volume (e.g., the peripheral zone of the prostate gland, anterior rectal wall, and prostatic urethra), in exactly the same position as they are during the intervention. D'Amico et al. reported the clinical efficiency of MRI-guided prostate brachytherapy using a $0.5 \mathrm{~T}$ openconfiguration MRI scanner 12]. The clinician approaches the patient in the scanner gantry and inserts multiple needles to into the lesion by using a grid of holes as a guide in a workflow similar to that of the traditional transrectal

D. Metaxas et al. (Eds.): MICCAI 2008, Part II, LNCS 5242, pp. 701709, 2008.

(C) Springer-Verlag Berlin Heidelberg 2008 
ultrasound-guided approach. Semi real-time MRI was obtained to confirm that the needle reached the target and was assessed by the medical physicist for dosimetric accuracy.

Although the open-configuration MRI provides easy access to the patient positioned inside the scanner, the clinical demands are changing towards higherspatial resolution and signal-to-noise ratio, leading to investigation of high-field closed-bore scanners. Susil et al. performed prostate biopsy and brachytherapy using a $1.5 \mathrm{~T}$ closed-bore MRI scanner by transferring the patient from the bore for insertions and back in for imaging [3]. Recently, 3T MRI has been available for diagnosis of prostate cancer, and its merits have been reported in 456. However, the application of high-field closed-bore MRI to prostate intervention is still limited, because of its limited access to the patient. Therefore, there is a strong need for MRI-compatible devices that enable precise placement inside the MRI scanner bore. Krieger et al. presented a 2-DOF passive, manually actuated mechanism to guide the biopsy needle to the prostate by transrectal approach 7. Zangos et al. reported the first cadaveric study results using a commercial MR-guided assistance system [8].

In this study we present the software strategy to assist a MRI-guided robotic prostate intervention. Our focused application is transperineal prostate biopsy and brachytherapy in $3 \mathrm{~T}$ MRI, which has been presented in our previous work 9. The newly presented software leads an operator through the clinical workflow, and guides a physician to insert the needle into the target lesion. The user interface and workflow management were designed based on thorough analysis of the clinical workflow for MRI-guided prostate intervention. The software also incorporates the fiducial-based robot-to-image registration method, which is crucial for both semi real-time MR imaging and positioning of the robot, initially developed in [10]. We evaluated registration accuracy of the robot and latency of semi real-time MR image transfer to test the feasibility of the proposed strategy.

The engineering contribution of this paper is the software strategy to provide intuitive navigation in closed-bore MRI. We present a workflow mechanism implemented in open-source image-guided therapy software, 3D Slicer. For intuitive needle insertion guidance, the key concept in this study is "closed-loop" therapy, which is the new clinical method where the robot's action is immediately captured in semi real-time MRI and instantaneous feedback is provided to a physician who decides about the next action. Therefore, the study is of clinical significance, by allowing the physician to monitor the needle in the tissue, and correct the insertion path and depth by using needle steering technique, such as [1], as necessary.

\section{Methods}

\subsection{System Architecture Overview}

The software system consists of three major components: 1) the control unit for the needle placement robot (Fig. 1A), 2) closed-bore whole body 3T MRI scanner (GE Excite HD 3T, GE Healthcare, Chalfont St. Giles, UK), and 3) open-source 
surgical navigation software (3D Slicer, http://www.slicer.org/) [12]. The 3D Slicer, running on a Linux-based workstation (SunJava Workstation W2100z, Sun Microsystems, CA), serves as a user interface for the entire system; it displays pre-operative $3 \mathrm{D}$ images for planning and intra-operative semi real-time MR images for guiding the procedure. The physician interactively specifies target points on the pre-operative image set; the current position is indicated on the $3 \mathrm{D}$ view of the $3 \mathrm{D}$ Slicer. All components were connected to one another via 100Base-T Ethernet. NaviTrack, an open-source library for device communication [13, is used to exchange various types of data, including commands to the robot and scanner, semi real-time images, and positional data (Fig. 1B). The positions of the target lesion are specified on the 3D Slicer interface and transferred to the robot control unit. While the robot control unit is driving the needle towards the target, the needle position is calculated from the optical encoders and sent back to the 3D Slicer every $100 \mathrm{~ms}$. The 3D Slicer calculates the imaging plane that intersects the needle's axis and transfers it to the scanner, which in turn acquires semi real-time images in that plane. Since all positional data exchanged among the components is represented in the image coordinate system, the robot coordinate system needs to be registered to the MR image coordinate system prior to the procedure.

\subsection{Workphase and Wizard Interface}

We defined six states of the system called "workphase," reflecting the six phases of clinical scenario of the prostate biopsy and brachytherapy using the robotic system: START-UP, PLANNING, CALIBRATION, TARGETING, MANUAL, and EMERGENCY. The workphase determines the behavior of the system that is required for each clinical phase. Each component switches its workphase according to a command received from the 3D Slicer; the phases of all components are always synchronized. Details of each workphase are described below:

- START-UP. Software and hardware systems are initialized in this workphase. The 3D Slicer reads a configuration file that defines data stream among the components (Fig. 1B). Meanwhile, the robot is set up by connecting the pneumatic system to pressurized air, connecting the device to the control unit, and attaching sterilized needle driver kit and needle to the robot. The needle is adjusted to a pre-defined home position of the robot. The imaging coil is attached to the patient, who is then positioned in the scanner.

- PLANNING. Pre-procedure 3D images, including T1- and T2-weighted images, are acquired and loaded into the 3D Slicer. Target points for needle insertions are interactively defined on the pre-operative images.

- CALIBRATION. The transformation that registers robot coordinates to patient coordinates is calculated by acquiring images of the Z-shape fiducial frame. The calibration procedure is performed for every intervention by the operator. Once the robot coordinate system is registered, the robot control unit and the 3D Slicer exchange target positions and the current position of 


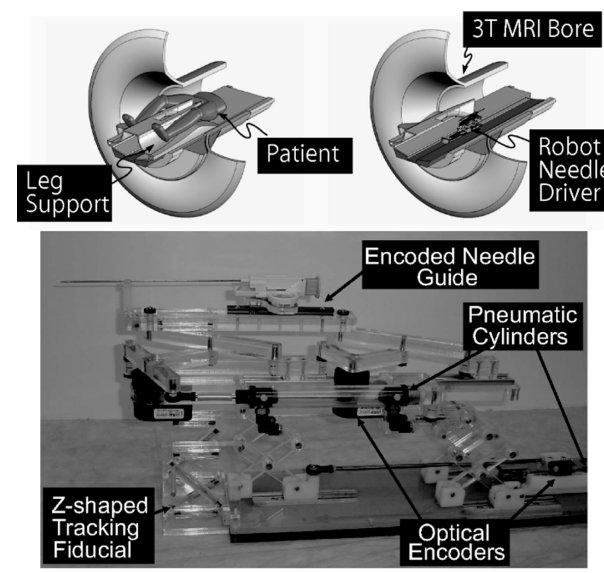

(A)

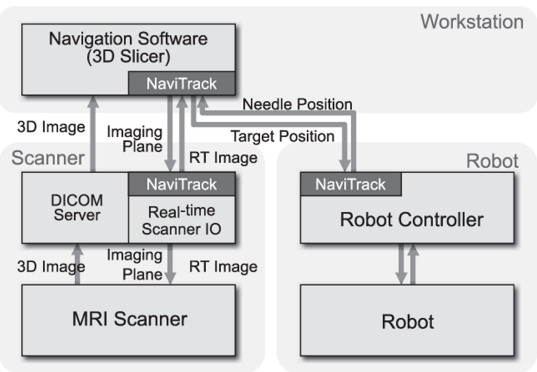

(B)

Fig. 1. (A) A robot for transperineal prostate biopsy and treatment 9]. Pneumatic actuators and optical encoders allow operating the robot inside a closed-bore 3T MRI scanner. Z-shape fiducial frame was attached for a calibration. (B) The diagram shows communication data flow of the proposed software system.

the needle using the image (i.e., patient) coordinate system. Details of the Z-shape fiducial will be described in Section 2.3.

- TARGETING. A current target is selected from the targets defined in the PLANNING workphase, and sent to the robot control unit. The robot moves the needle to the target while transmitting its current position in real time. After the needle guide is maneuvered to the desired position, the needle is manually inserted along an encoded guide to the target lesion. The insertion process is monitored through semi real-time 2D image, automatically aligned to the plane along the needle axis.

- MANUAL. The operator can directly control the robot position remotely from the 3D Slicer. The system enters this workphase when the needle position needs to be adjusted manually.

- EMERGENCY. All robot motion is halted for safety reasons, as soon as the system enters the EMERGENCY workphase. The actuators are locked in this state to prevent unwanted motion and allow manual needle retraction.

The transitions between workphases are invoked by the operator using an interactive graphical user interface (GUI) of the 3D Slicer. To offer intuitive operation, a wizard-style interface is employed for the GUI (Fig. 2). Each page in the wizard corresponds to a workphase to hide unavailable functions from the operator.

\subsection{Registration}

To transform a position from the image coordinate system to the robot coordinate system, or vice versa, a calibration is performed by registering the Z-shape fiducial frame [10] attached to the robot in the image coordinate system. The 


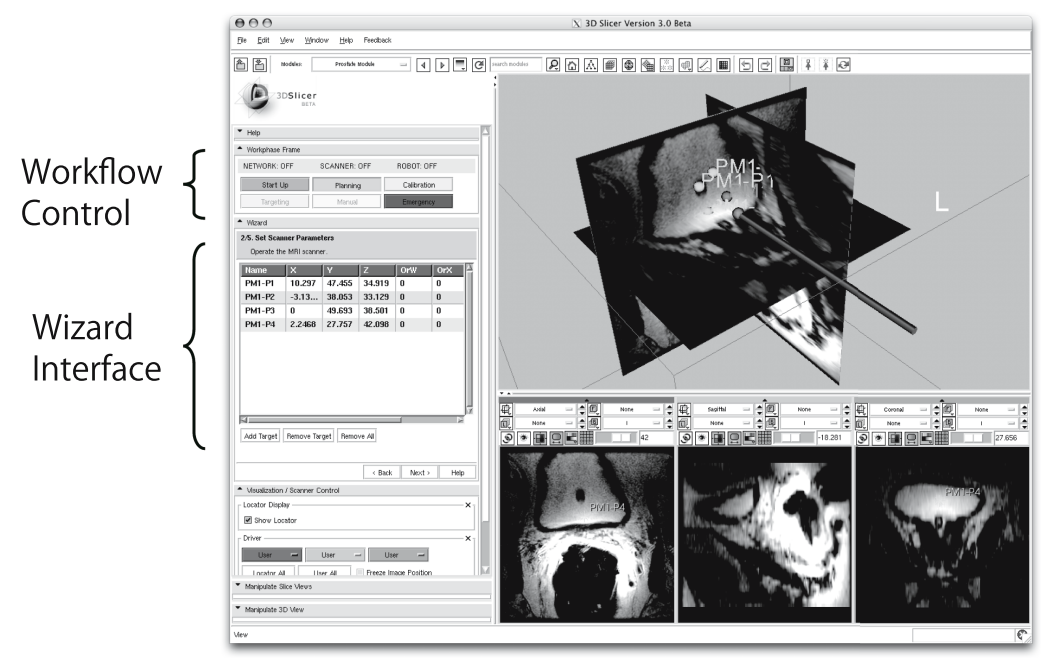

Fig. 2. Screen shot of the graphical user interface (GUI) implemented in the surgical navigation software, 3D Slicer, is shown. Each step of the clinical workflow, or "workphase," is assigned to each page of the wizard interface, which navigates operator to follow the procedure. The GUI also incorporates buttons to directly jump to other workphases.

Z-shape fiducial frame is designed to be localized from a single 2D image intersecting the frame. The position and orientation of the Z-shape fiducial frame provide the transformation between the image and robot coordinate systems. Once the transformation is calculated, the needle position and orientation measured by the encoders of the robot can be transformed to image coordinates. The rigid structure of the fiducial frame is made up of seven rigid glass tubes with $3 \mathrm{~mm}$ inner diameters that are filled with contrast agent (MR Spots, Beekley, Bristol, CT) and placed on three faces of a $60 \mathrm{~mm}$ cube. In this study, a fast spoiled gradient echo recalled (SPGR) sequence was employed with time of repetition (TR)/ echo time (TE): $34 / 3.7 \mathrm{~ms}$, flip: 30 degree, number of excitation (NEX): 3, field of view (FOV): $160 \mathrm{~mm}$ to locate the fiducial frame.

\section{Experiments}

The calibration accuracy of the Z-shape fiducial, positional accuracy of the imaged target, and latency of the image display are evaluated using a phantom to test the feasibility of the system. In this study, the robot control unit was replaced by a software simulator, which receives the target position and returns exactly the same position to the 3D Slicer as a current virtual needle position. The reason to use the simulator is that it excludes any mechanical error from the robot. The orientation of the virtual needle was fixed along the static magnetic 


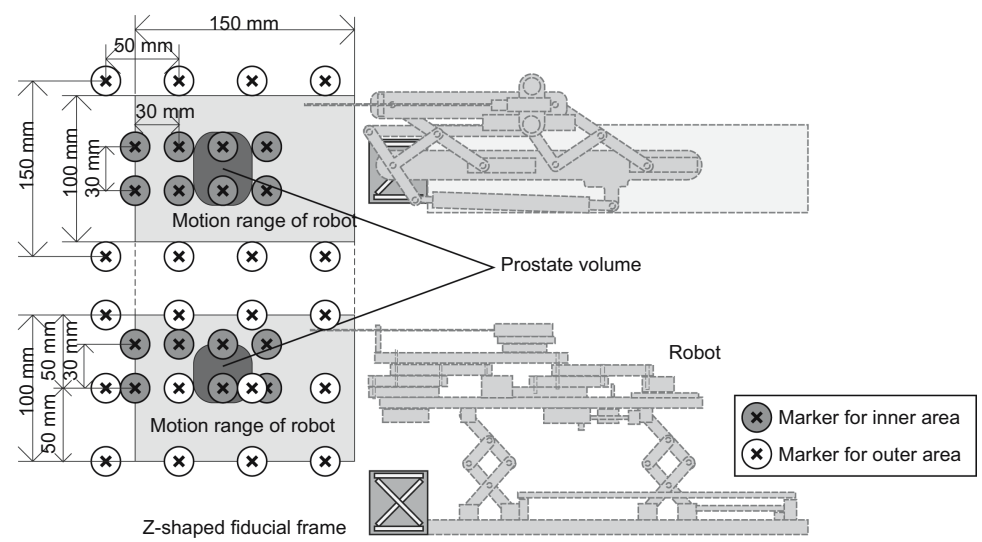

Fig. 3. The physical relationship among robot, z-frame, targets, range of motion, and markers is shown. The Z-shape fiducial frame is attached. Twenty-four markers are in the outer area of the phantom, which covers entire motion range of the robot, and 16 markers are in the inner area, covering the typical position of the prostate.

field to simulate the transperineal biopsy/brachytherapy case, where the needle approaches from inferior to superior direction. The phantom is made up of 24 markers embedded at known positions in outer area of the volume and 16 markers in inner area of the volume (corresponding to the end of the motion range of the robot and the typical position of the prostate, respectively), and was placed in the gantry of the scanner (Fig. 3). The Z-shape frame was precisely affixed to the phantom instead of the robot to register the marker positions to the MR image coordinates. The physical relationship between the target markers and the Z-shape frame was approximately the same as the clinical case.

To evaluate accuracy, each marker position was calculated based on the position/orientation of the Z-shape fiducial frame determined by the intersecting $2 \mathrm{D}$ image, and known physical location of each marker with respect to the fiducial frame (simulating robot encoder output). A 3D image was acquired as a gold standard, and imaged and registered marker positions were compared. The parameters for the $3 \mathrm{D}$ imaging were: matrix $=256 \times 256 \times 72 ; \mathrm{FOV}=30 \mathrm{~mm}$; slice thickness $=2 \mathrm{~mm} ; \mathrm{TR} / \mathrm{TE}=6.3 / 2.1 \mathrm{~ms}$; flip angle $=30 \mathrm{deg}$.

The accuracy of the position of imaged targets on $2 \mathrm{D}$ semi real-time images was also evaluated. A 2D spoiled gradient recalled (SPGR) sequence was used with following parameters: matrix $=256 \times 256$; FOV $=30 \mathrm{~mm}$; slice thickness $=$ $5 \mathrm{~mm}$; flip angle $=30 \mathrm{deg}$. To vary the frame rate, TR/TE were set to $12.8 / 6.2 \mathrm{~ms}$ (3.3 s/frame), 15.2/5.1ms (3.9 s/frame), and 18.6/5.0ms (4.8 s/frame). To quantify the position error of the $2 \mathrm{D}$ images, each marker was specified as the virtual needle tip, and three orthogonal planes were acquired. Positional error of imaging plane was defined as the offset of the imaged target marker from the intersection of the three planes.

In addition, semi real-time imaging latency was evaluated by oscillating the position of the virtual needle tip with a range of $100 \mathrm{~mm}$ and period of $20 \mathrm{~s}$ 
during imaging the phantom. One hundred twenty images were recorded with time stamps for each frame. Since the center of the image follows the virtual needle tip motion and the imaged phantom shifted on the semi real-time image frame by frame, the actual time points of image acquisition were quantified by measuring the shift of the phantom image.

Semi real-time 2D images were successfully acquired in three orthogonal planes parallel and perpendicular to the simulated needle axis, and visualized on the navigation software. The root mean square (RMS) of positional error due to the calibration was $4.8 \mathrm{~mm}$ for outer area and $2.6 \mathrm{~mm}$ for inner area (representing the area within the prostate capsule). In the semi real-time images, the RMS error between the specified target position and imaged target was $3.7 \mathrm{~mm}$ for the targets in the outer area and $1.8 \mathrm{~mm}$ for the inner area. The latencies of displaying the semi real-time image on the navigation software for each frame rate was $5.0 \mathrm{~s}(3.3 \mathrm{~s} /$ frame), $5.6 \mathrm{~s}(3.9 \mathrm{~s} /$ frame), and $6.5 \mathrm{~s}$ (4.8 s/frame), since the navigation software received the needle position from the robot. Standard deviations of the latencies were $0.06-0.07 \mathrm{~s}$ in all cases.

\section{Discussion and Conclusion}

Design and implementation of a navigation system for robotic transperineal prostate therapy in closed-bore MRI is presented. The clinical workflow is separated into the six "workphases" to define the behavior of the system based on the stage of the treatment process. Each workphase is associated with each page of the wizard interface, which navigates the operator to follow the clinical workflow easily and intuitively. The workphases are defined based on the clinical workflow of MRI-guided robotic transperineal prostate biopsy and brachytherapy, but are consistent with most MRI-guided robotic intervention (e.g., liver ablation therapy 14 ).

The proposed software system incorporates a calibration based on the Z-shape fiducial frame to register the robot coordinate system to the image coordinate system. The accuracy study demonstrates that the integrated system provided sufficient registration accuracy for prostate biopsy and brachytherapy compared with the clinical significant size $(0.5 \mathrm{cc})$ and traditional grid spacing $(5 \mathrm{~mm})$. The result is also comparable with the accuracy study on clinical targeted biopsy by Blumenfeld et al. $(6.5 \mathrm{~mm})$ [15]. Since mechanical error was excluded from the condition in the presented report, the study must be continued to evaluate the overall accuracy of targeting using the robotic device guided by the system. It was reported that the RMS positioning error due to the mechanism was 0.94 $\mathrm{mm}$ 9.

The study also demonstrates that the semi real-time 2D MR images captured the target with sufficient positional accuracy and reasonable latency. The accuracy was degraded in the outer area of the phantom, where the distance from the Z-shape frame was longer than the inner area. In addition, the image was distorted by the field inhomogeneity, causing positional error of the imaged target. Thus, a distortion correction after image reconstruction could be effective 
in improving accuracy. The frame rate and latency of the semi real-time image is also essential for interactivity during guidance. It is possible to decrease the latency to approx. $3 \mathrm{~s}$ by using a faster imaging sequence with frame rate of near $1 \mathrm{frame} / \mathrm{s}$, since the latency study indicates that the latency due to the system (data transfer and image reconstruction) was $1.7 \mathrm{~s}$.

In conclusion, the proposed system provides a user interface based upon the workphase concept that allows operators intuitively to walk through the clinical workflow. It is demonstrated that the system provides semi real-time image guidance with adequate accuracy and speed for interactive needle insertion in MRI-guided robotic intervention for prostate thereapy.

Acknowledgments. This work is supported by 1R01CA111288, 5U41RR019703, 5P01CA067165, 1R01CA124377, 5P41RR013218, 5U54EB005149, 5R01CA109246 from NIH. Its contents are solely the responsibility of the authors and do not necessarily represent the official views of the NIH. This study was also in part supported by NSF 9731748, CIMIT, Intelligent Surgical Instruments Project of METI (Japan).

\section{References}

1. D'Amico, A.V., Cormack, R., Tempany, C.M., et al.: Real-time magnetic resonance image-guided interstitial brachytherapy in the treatment of select patients with clinically localized prostate cancer. Int. J. Radiat. Oncol. Biol. Phys. 42(3), 507515 (1998)

2. D'Amico, A.V., Tempany, C.M., Cormack, R., others,: Transperineal magnetic resonance image guided prostate biopsy. J. Urol. 164(2), 385-387 (2000)

3. Susil, R., Camphausen, K., Choyke, P., et al.: System for prostate brachytherapy and biopsy in a standard 1.5 T MRI scanner. Magn. Reson. Med. 52(3), 683-687 (2004)

4. Sosna, J., Pedrosa, I., Dewolf, W., et al.: MR imaging of the prostate at 3 tesla: comparison of an external phased-array coil to imaging with an endorectal coil at 1.5 tesla. Acad. Radiol. 11(8), 857-862 (2004)

5. Bloch, B., Rofsky, N., Baroni, R., et al.: 3 tesla magnetic resonance imaging of the prostate with combined pelvic phased-array and endorectal coils; initial experience(1). Acad. Radiol. 11(8), 863-867 (2004)

6. Futterer, J., Scheenen, T., Huisman, H.: Initial experience of 3 tesla endorectal coil magnetic resonance imaging and $1 \mathrm{~h}$-spectroscopic imaging of the prostate. Invest. Radiol. 39(11), 671-680 (2004)

7. Krieger, A., Susil, R.C., Menard, C., et al.: Design of a novel MRI compatible manipulator for image guided prostate interventions. In: IEEE TBME, February 2005, vol. 52, pp. 306-313 (2005)

8. Zangos, S., Eichler, K., Engelmann, K., et al.: MR-guided transgluteal biopsies with an open low-field system in patients with clinically suspected prostate cancer: technique and preliminary results. Eur. Radiol. 15(1), 174-182 (2005)

9. Fischer, G.S., Iordachita, I., Csoma, C., et al.: MRI-Compatible Pneumatic Robot for Transperineal Prostate Needle Placement. IEEE/ASME Trans. Mechatronics 13(3), 295-305 (2008) 
10. DiMaio, S., Samset, E., Fischer, G., et al.: Dynamic MRI scan plane control for passive tracking of instruments and devices. In: Ayache, N., Ourselin, S., Maeder, A. (eds.) MICCAI 2007, Part II. LNCS, vol. 4792, pp. 50-58. Springer, Heidelberg (2007)

11. DiMaio, S.P., Salcudean, S.: Needle steering and motion planning in soft tissues. IEEE Trans. Biomed. Eng. 52(6), 965-974 (2005)

12. Gering, D.T., Nabavi, A., Kikinis, R., et al.: An integrated visualization system for surgical planning and guidance using image fusion and an open MR. J. Magn. Reson. Imag. 13(6), 967-975 (2001)

13. Spiczak, J.V., Samset, E., Dimaio, S., et al.: Device connectivity for image-guided medical applications. Stud. Health Technol. Inform. 125 (2007)

14. Morikawa, S., Inubushi, T., Kurumi, Y., et al.: MR-guided microwave thermocoagulation therapy of liver tumors: Initial clinical experiences using a $0.5 \mathrm{~T}$ open MR system. Journal of Magnetic Resonance Imaging 16(5), 576-583 (2002)

15. Blumenfeld, P., Hata, N., DiMaio, S., et al.: Transperineal prostate biopsy under magnetic resonance image guidance: a needle placement accuracy study. J. Magn. Reson. Imaging 26(3), 688-694 (2007) 